\title{
HEAT-TRANSFER ENHANCEMENT BY ADAPTIVE REORIENTATION OF FLOW FIELDS
}

\author{
R. Lensvelt*, M.F.M. Speetjens, H. Nijmeijer \\ Department of Mechanical Engineering, Eindhoven University of Technology, Eindhoven, PO Box 513, \\ 5600 MB, The Netherlands
}

\begin{abstract}
Scope is enhancement of scalar transport (heat, chemical species) in engineered flow systems by reorientations of a laminar base flow. Practical applications include mixing in inline heat exchangers by downstream reorientation of baffles, stirring in bio-reactors by cyclic repositioning of impellers, and subsurface chemicals distribution for in situ minerals mining by unsteady pumping schemes. Conventional reorientation schemes consist of a periodic reorientation (in space or time) of the flow designed to accomplish efficient fluid mixing. However, whether this approach indeed yields optimal scalar transport for significant diffusion and/or chemical reactions is unclear. The present study explores an alternative approach: adaptive reorientation of the flow by interval-wise selection of the reorientation that is predicted to yield optimal scalar transport for a future time horizon. Key enabler for fast predictions is a compact model based on the spectral decomposition of the scalar evolution in the base flow. The adaptive reorientation scheme is investigated for a representative problem: enhanced heating of a cold fluid in a $2 D$ circular domain by an unsteady flow driven by step-wise activation of moving boundary segments. This reveals that the adaptive reorientation scheme can substantially accelerate the heating compared to conventional time-periodic reorientation designed for efficient mixing and thus demonstrates its potential for attaining optimal scalar transport in reoriented flows.
\end{abstract}

KEY WORDS: chaotic advection, scalar transport, mixing

\section{INTRODUCTION}

The enhancement of scalar transport finds its immediate application in, for example, industrial mixing and heat-transfer processes where promotion of scalar redistribution serves to improve mixing/heating rate [1]. Industries involving subsurface flows to, for example, extract geothermal energy or minerals through production fluid redistribution [2], dissolution of subsurface minerals or groundwater remediation [3], or to accomplish subsurface resource confinement (e.g. pollutants) [4 can greatly benefit from enhanced redistribution of scalar quantities. The required redistribution can be accomplished through laminar base flows that follow well-known principles to promote mixing set forth in [5]. Key features in the applications of interest are that scalar transport is "driven" by i) temperature gradients due to heat transport across system boundaries and/or ii) the presence of significant diffusion/chemical reactions. Conventional remediation/heating approaches employ reorientation schemes (periodic in space or time) specifically designed for efficient fluid mixing. Rationale for this methodology is that effective exploitation of the stretch-and-fold mechanism underpinning mixing automatically yields optimal heat transfer. Despite its validity in the advective limit this assumption does not necessarily hold for the 
intended applications for at least two reasons. First, heat transport by diffusion renders the link between fluid motion and thermal transport highly non-trivial. Second, heat transport across the system boundary relies on temperature gradients to sufficiently "drive" transfer and thus, inherently, requires a heterogeneous scalar field. However, efficient fluid mixing relies on the rapid homogenization of the scalar field. This strongly suggests that efficient fluid mixing schemes may, at least in the vicinity of the boundary, create a sub-optimal or even counter-productive condition for accelerated heat transfer. Recent developments have focussed on the application of optimal control to develop reorientation schemes with feedback that further accelerates homogenization [6]. However, the developed reorientation scheme is designed for (sub)optimal fluid mixing which only serves limited utility in the present study for above mentioned reasons. Nonetheless, the generic approach permits a generalisation towards thermal problems (i.e. transport including significant diffusion) and serves, without loss of generality, as a bases for the adaptive reorientation scheme proposed in this study. The remainder of this work presents a novel approach according to the following structure. In Sec. 2, we present the planar configuration of an inline mixer that, together with the governing equations, describes the mechanism of interest for enhancing heat transport. This system description is then used to present a case where transport enhancement is limited for the conventional time-periodic approach in Sec. 3. Next, we detail the theoretical framework underpinning the novel approach and show its capacity to accelerate heating in Sec. 4. Finally, conclusions and recommendations for future research are presented in Sec. 5.

\section{PROBLEM FORMULATION AND NUMERICAL METHOD}

\subsection{Configuration}

This work adopts the 2D unsteady Rotated Arc Mixer (RAM) introduced in Ref. [7] as a representative configuration for an in-depth transport analysis (Fig. 1a). The $2 D$ RAM consists of a circular domain $\mathcal{D}=\left\{(r, \theta) \in \mathbb{R}^{2} \mid r \leq R,-\pi \leq \theta \leq \pi\right\}$ with radius $R$ which is enclosed by a boundary $\Gamma=\partial \mathcal{D}$ as shown in Fig. 1. It heats an initially cold fluid inside $\mathcal{D}$ through a constant temperature at the circumference $\Gamma$. Circumference $\Gamma$ contains $N$ apertures of arc length $\Delta$ which consecutively differ from each other

(a) Geometry

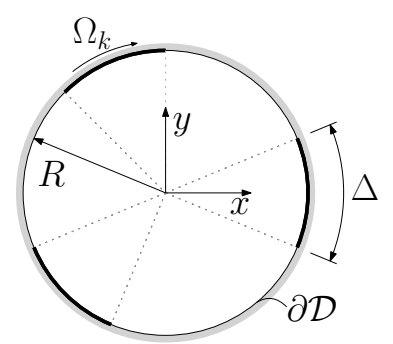

(b) Aperture 1

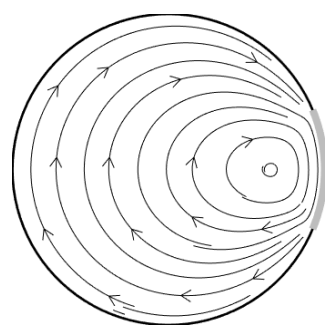

(c) Aperture 2

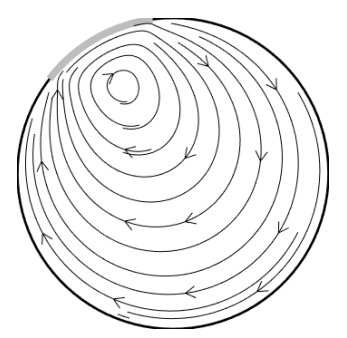

(d) Aperture 3

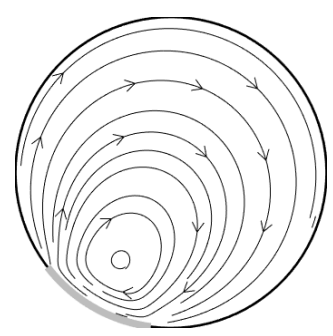

Figure 1 Geometry of $2 D$ Rotating Arc Mixer (RAM) with three apertures $(N=3)$ of arc length $\Delta=\pi / 4$ and reoriented streamlines corresponding to activate aperture in (b)-(d).

by an angular offset $\Theta=2 \pi / N$. These aperture activations serve to influence heat transport through advection such that homogenization of the scalar field inside $\mathcal{D}$ accelerates. The geometric parameters throughout this study are fixed to $N=3$ and $\Delta=\pi / 4$, as shown in Fig. 1a, which can be done without loss of generality. No-slip boundary conditions assure the absence of flow at the circumference $\Gamma$ except at an aperture $k$ where only the angular velocity $\omega_{k}$ drives the fluid flow inside $\mathcal{D}$ through viscous drag. These conditions can be physically realized through a motor-belt system as presented in Baskan et al. [7]. Laminar flow conditions give apertures an associated steady field as illustrated by streamlines of the base flow in Fig. 1b. Note that in the present study individual aperture activation results in reorientation of the base flow by $\Theta=2 \pi / N$ as shown in Fig. 1b-Fig. 1d. Exact implementation of the flow model for the $2 D$ RAM is detailed in Sec. 2.2. Transport with multiple apertures requires a 
proper activation order to significantly accelerate homogenization compared to mere diffusive heating. Therefore, the aim of this work is to devise an aperture activation scheme such that the transient for heating the initially cold fluid to the boundary temperature is minimized.

\subsection{Governing equations}

Fluid motion is governed by the conservation laws for mass and momentum, which, assuming incompressibility, laminar flow and absence of body forces other than gravity, in non-dimensional form read

$$
\boldsymbol{\nabla} \cdot \mathbf{v}=0, \quad \operatorname{Sr} \frac{\partial \mathbf{v}}{\partial t}+\operatorname{Re} \mathbf{v} \cdot \nabla \mathbf{v}=-\nabla P+\nabla^{2} \mathbf{v}
$$

with $\mathbf{v}(\mathbf{x}, t)$ the fluid velocity and $P(\mathbf{x}, t)$ the pressure in position $\mathbf{x} \in \mathcal{D}$ at time $t \geq 0$. The corresponding initial and boundary conditions are given by

$$
\mathbf{v}(\mathbf{x}, 0)=\mathbf{0}, \quad \forall \mathbf{x} \in \mathcal{D}, \quad \text { and } \quad \mathbf{v}(\mathbf{x}, t)=\left\{\begin{array}{cc}
\omega \mathbf{e}_{\theta} & \text { if } \mathbf{x} \in \Gamma_{k} \\
\mathbf{0} & \text { if } \mathbf{x} \in \Gamma \backslash \Gamma_{k}
\end{array}\right.
$$

where $\Gamma_{k}=\{(r, \theta) \in \Gamma \mid-\Delta / 2 \leq \theta-(k-1) \Theta \leq \Delta / 2\}$ and $\mathbf{e}_{\theta}$ indicate aperture $k$ and the unit vector in azimuthal direction, respectively. Coefficient $\omega= \pm 1$ determines the sense of rotation: clockwise $(\omega=1)$ versus counter-clockwise $(\omega=-1)$. System (1) is parameterized by the Strouhal number $S r=\tau_{v} / \tau=R^{2} / v \tau$ and the Reynolds number $R e=U R / v=\Omega R^{2} / v$, with $\tau_{v}=R^{2} / v$ the viscous time scale, $U=\Omega R$ the azimuthal velocity of the apertures, $v$ the kinematic viscosity of the fluid, $R$ as in Fig. $1 \mathrm{a}$ and $\tau$ the aperture activation time. Strongly laminar conditions imply (i) a rapid response of the fluid to changes in flow forcing by the apertures (i.e. $S r=\tau_{v} / \tau \ll 1$ ) and (ii) negligible fluid inertia (i.e. $R e \ll 1$ ). As presented in Baskan et al. [7] this simplifies (1] to

$$
\boldsymbol{\nabla} \cdot \mathbf{v}=0, \quad-\nabla P+\nabla^{2} \mathbf{v}=\mathbf{0},
$$

which with boundary conditions (2) describes aperture-wise steady Stokes flow that admits an analytical expression for each aperture following Ref. [8]. Absence of explicit time dependence in (3) means that unsteadiness enters the fluid motion entirely through switching between apertures $k$ via boundary condition (2). Moreover, simplification to steady Stokes flows causes the aperture reorientations to carry over to the flow, meaning that flow $\mathbf{v}_{k}$ driven by aperture $k$ is just a reorientation of the base flow $\mathbf{v}_{1}$ according to

$$
\mathbf{v}_{k}(\mathbf{x})=\mathbf{v}_{k}(r, \theta)=\mathbf{v}_{1}(r, \theta+(k-1) \Theta)=\mathbf{v}_{1}\left(\mathcal{R}_{k}(\mathbf{x})\right)
$$

as illustrated in Fig. 1b-Fig. $1 \mathrm{~d}$ by the streamline patterns for the $N=3$ apertures considered in the present study. The class of unsteady flows investigated hereafter are constructed from reorientations of these aperture-wise steady flows in (4) following

$$
\mathbf{v}(\mathbf{x}, t)=\mathbf{v}_{u(t)}(\mathbf{x}), \quad u(t)=\sum_{n=0}^{\infty} u_{n}\left(H\left(t-t_{n+1}\right)-H\left(t-t_{n}\right)\right),
$$

with $u(t)$ the "orientation scheme" that activates aperture $u_{n} \in \mathcal{U}=\left\{u_{0}, u_{1}, \ldots\right\}$ and the corresponding flow $\mathbf{v}_{k}$ according to Fig. 1 during time interval $t_{n} \leq t \leq t_{n+1}$. Here $H(t)$ is the well-known Heaviside function and $t_{n}=n \tau$. Set $\mathcal{U}$ determines the particular aperture sequence and thus the time signature of the flow; e.g. $\mathcal{U}=\{1,3,2\}$ subsequently activates apertures $(1,3,2)$ each for a duration $\tau$.

\subsection{Heat transfer}

Heat transfer is governed by the conservation law for energy, which for the flow conditions in the RAM identifies with the advection-diffusion equation for the temperature field $T(\mathbf{x}, t)$, reading

$$
\frac{\partial T}{\partial t}=-\mathbf{v} \cdot \nabla T+\frac{1}{P e} \nabla^{2} T
$$


with $\mathbf{v}(\mathbf{x}, t)$ the unsteady flow according to (5), $P e=U R / \mathrm{k}$ the Péclet number and $\mathrm{k}$ the thermal diffusivity. The uniform initial and boundary conditions for the heating problem defined in Sec. 2.1 are

$$
T(\mathbf{x}, 0)=T_{0}, \quad \forall \mathbf{x} \in \mathcal{D}, \quad \text { and }\left.\quad T(\mathbf{x}, 0)\right|_{\Gamma}=T_{\infty},
$$

with $T_{0}$ the cold initial fluid temperature and $T_{\infty}>T_{0}$ the hot boundary temperature. In practical systems typically $P e \sim \mathcal{O}\left(10^{3}\right)$, implying advection-dominated heat transfer yet still with a significant contribution by diffusion. Heat transfer governed by (6) can (without loss of generality) be expressed in terms of the transient temperature $\widetilde{T}(\mathbf{x}, t)$ according to

$$
\widetilde{T}(\mathbf{x}, t) \equiv T(\mathbf{x}, t)-T_{\infty},
$$

using property $\lim _{t \rightarrow \infty} T(\mathbf{x}, t)=T_{\infty}$ for arbitrary $\mathbf{v}$ in present uniform case $T_{\infty}$. Transient $\widetilde{T}$ allows an investigation of heat transfer relative to a reference $T_{\infty}$ analogous to an error analysis encountered in control theory Ref. [9]. This motivates the adaptive scheme based on transient temperature in Sec. 4.

\subsection{Numerical method}

Numerical simulation of the RAM employs the dedicated method proposed by Ref. [10]. This hinges on a semi-discrete model obtained by discretization of (6) for each steady flow $\mathbf{v}_{k}$, yielding

$$
\frac{d \widetilde{\mathbf{T}}(t)}{d t}=\mathbf{A}_{k} \widetilde{\mathbf{T}}(t)
$$

with $\widetilde{\mathbf{T}}(t)=\left[\widetilde{T}\left(\mathbf{x}_{0}, t\right), \ldots, \widetilde{T}\left(\mathbf{x}_{M}, t\right)\right]^{\dagger}$ the nodal temperature value vector on computational grid $\mathbf{X}=$ $\left[\mathbf{x}_{0}, \ldots, \mathbf{x}_{M}\right]^{\dagger}$ and $\mathbf{A}_{k}$ the discrete matrix approximation of the advection-diffusion operator ( $\dagger$ indicates transpose). Time-independence of $\mathbf{v}_{k}$ implies a time-invariant $\mathbf{A}_{k}$ and thus admits

$$
\widetilde{\mathbf{T}}(t)=\mathbf{U}_{k} \widetilde{\mathbf{T}}_{0}, \quad \mathbf{U}_{k}=\mathbf{V}_{k} e^{\boldsymbol{\Lambda}_{k} t} \mathbf{V}_{k}^{-1},
$$

as semi-analytical solution for (9) using the spectral decomposition $\mathbf{A}_{k}=\mathbf{V}_{k} \Lambda_{k} \mathbf{V}_{k}$, with $\mathbf{V}_{k}=$ $\left[\boldsymbol{\phi}_{0}^{(k)}, \ldots, \boldsymbol{\phi}_{M}^{(k)}\right]$ and $\boldsymbol{\Lambda}_{k}=\operatorname{diag}\left(\lambda_{0}^{(k)}, \ldots, \lambda_{M}^{(k)}\right)$ the standard eigenvector and eigenvalue matrices, respectively, of system matrix $\mathbf{A}_{k}$. Semi-discrete model (9) and its solution (10) are key to the (computational) methodology below for any orientation scheme $u(t)$ and resulting velocity $\mathbf{v}(\mathbf{x}, t)$ following (5). Note that conventional FDM/FVM/FEM discretizations essentially yield model structures similar to (9) such that the adaptive reorientation scheme, proposed in Sec. 4 , is generally applicable.

\section{CONVENTIONAL APPROACH: TIME-PERIODIC REORIENTATION}

To study the influence of fluid motion on homogenization a time-periodic reorientation scheme is employed below. These time-periodic flow reorientations substantially accelerate heating compared to diffusion-only heat transfer. A scheme is defined through a repeated subset $\mathcal{U}_{\mathcal{T}}=\{1, \ldots, N\}$ resulting in the time-periodic orientation scheme $\mathcal{U}=\left\{\mathcal{U}_{\mathcal{T}}, \mathcal{U}_{\mathcal{T}}, \ldots\right\}$. Via (5) this yields a flow field as

$$
\mathbf{v}\left(\mathbf{x}, t_{n}\right)=\mathbf{v}\left(\mathbf{x}, t_{n}+\mathcal{T}\right)
$$

with $\mathcal{T}=N \tau$ the period time. Aperture activation time, for $\mathcal{U}_{\mathcal{T}}=\{1,2,3\}$, is investigated by weighting transient temperature $\widetilde{\widetilde{T}}(t)$ on $\mathcal{D}$. Chaotic trajectories of tracers for these times imply both schemes mix effectively. However, Fig. 2 shows that heating rate (marginally) accelerates for increase in activation times. Fig. 3 shows temperature snapshots for the reorientation schemes with different $\tau$. In this study all temperatures are normalized between $\min (T(\mathbf{x}, t))=0$ (blue) and $\max (T(\mathbf{x}, t))=1$ (red). A comparison of these snapshots reveals vastly different temperature fields yet with similar heating rates. This implies that entirely different mixing flows have a comparable impact on the heat transfer and 


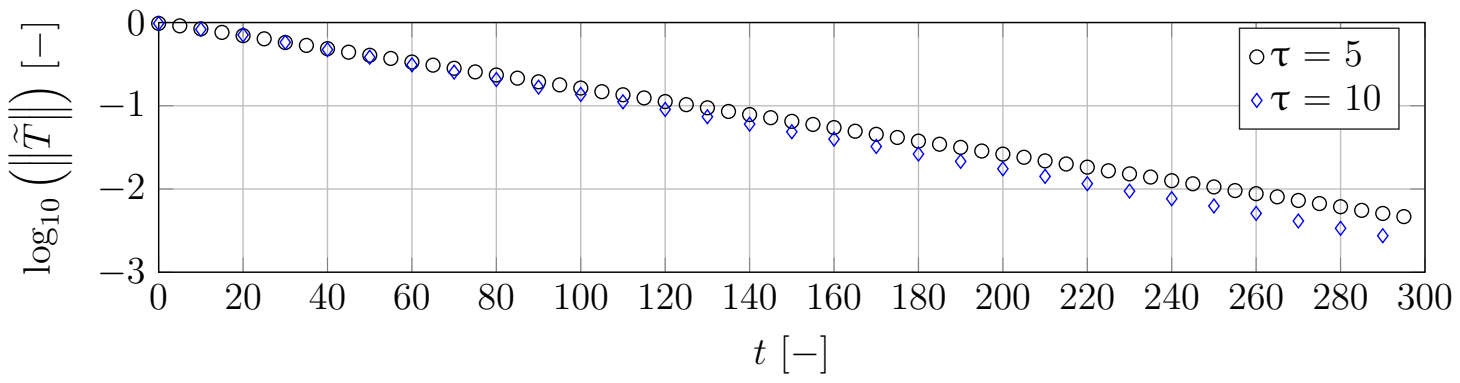

Figure 2 Comparison of the error field in advection dominated transport $\left(P e=10^{3}\right)$ for time-periodic orientation schemes with different aperture activation times.

optimization on the basis of mixing performance thus is an unreliable approach to determine the optimal conditions for heat transfer. Systematic enhancement of heat-transfer beyond time-periodic flow
(a) $t_{n}=0.1$.
(b) $t_{n}=10$.
(c) $t_{n}=20$.
(d) $t_{n}=30$.
(e) $t_{n}=50$.
(f) $t_{n}=100$.
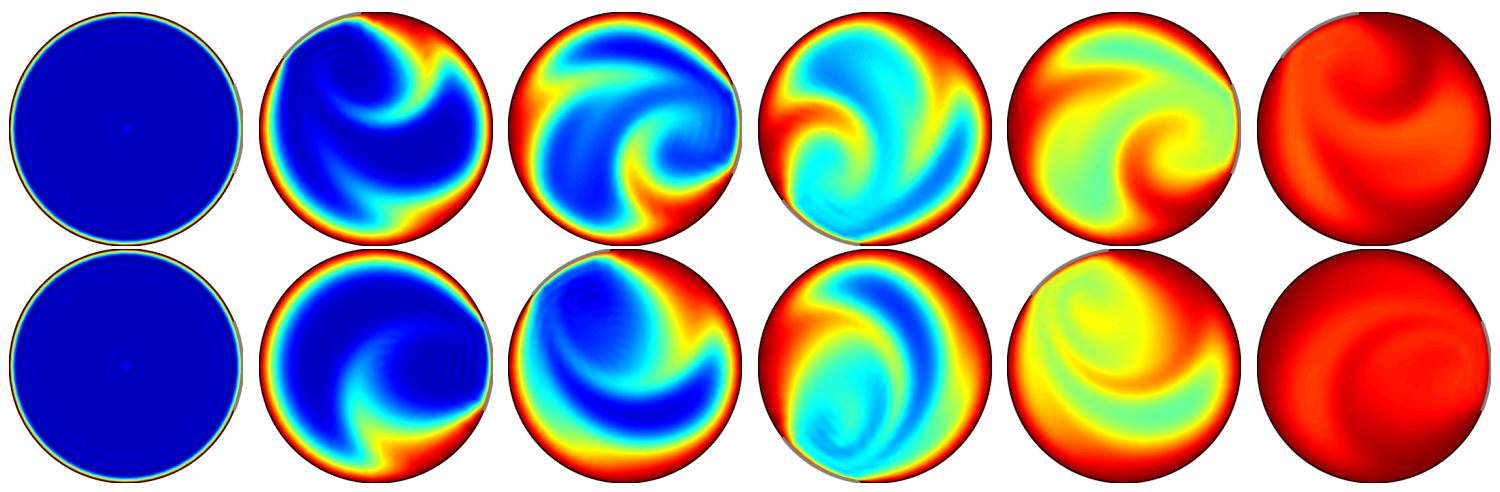

Figure 3 Snapshots of temperature evolution $T(\mathbf{x}, t)$ in an advection dominated flow $\left(P e=10^{3}\right)$ for time-periodic orientation schemes with aperture activation time $\tau=5$ (top row) and $\tau=10$ (bottom row).

reorientations designed for effective mixing thus requires a scheme directly based on the temperature field.

\section{NOVEL APPROACH: ADAPTIVE REORIENTATION}

\subsection{Control strategy}

Goal of the adaptive reorientation scheme proposed in this study is to minimize the transient from the initial temperature $T_{0}$ to the target temperature $T_{\infty}$. Therefore, a feedback controller is incorporated in the system that, on the basis of intermediate temperature fields, adapts the flow reorientation described by orientation scheme $u(t)$ following (5) such that it maximizes heating rate (Fig. 4). The procedure involves repeated execution of the following steps at each time step $t_{n}=n \tau$ :

- Step 1: predict the transient temperature $\widetilde{T}_{k}\left(\mathbf{x}, t_{n+1}\right)$ at time step $t_{n+1}$ for each aperture $k$ with transient temperature $\widetilde{T}\left(\mathbf{x}, t_{n}\right)$ at the current time-step $t_{n}$ as initial condition.

- Step 2: select the aperture $k$ that maximizes heating rate in time interval $t_{n} \leq t \leq t_{n+1}$ and set $u_{n}=k$ in aperture sequence $\mathcal{U}$ for the orientation scheme $u(t)$.

- Step 3: simulate the corresponding temperature evolution for time interval $t_{n} \leq t \leq t_{n+1}$. 
This procedure repeats each time step $t_{n}$ and attains maximal heating rates by minimizing

$$
\arg \min _{k} J_{k}\left(t_{n+1}\right), \quad J_{k}(t)=\int_{\mathcal{D}} \widetilde{T}_{k}^{2}(\mathbf{x}, t) d^{2} \mathbf{x},
$$

in which the cost-to-go $J_{k}$ represents the error between predicted and target temperature for $t \geq 0$. Practicality of the approach dictates that the aperture-wise transient temperatures predictions $\left(\widetilde{T}_{k}(\mathbf{x}, t)\right)$ in step 1 must be much faster than the aperture activation time $\tau$. To this end a compact model is proposed below.

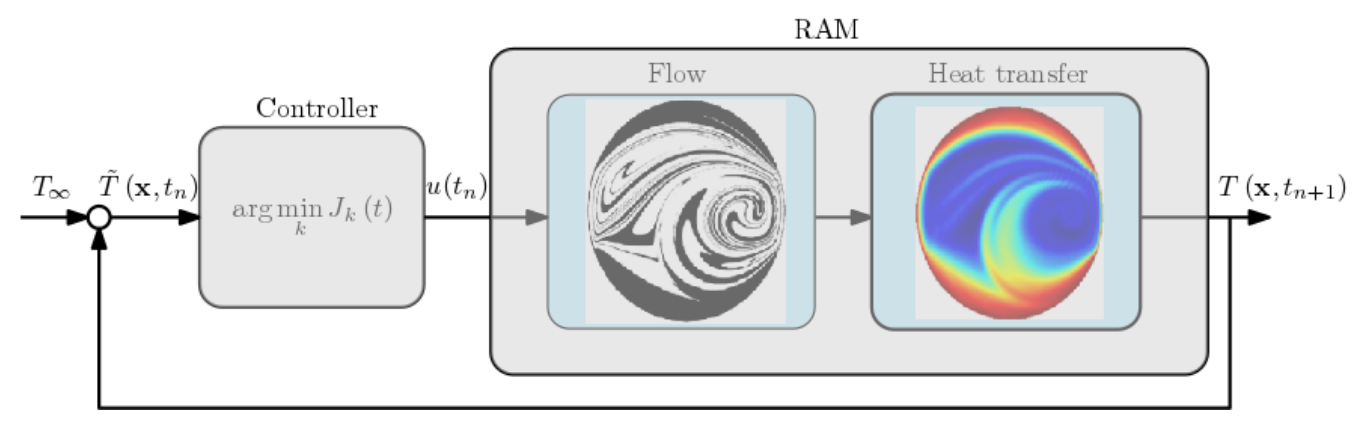

Figure 4 The closed loop for adaptive flow reorientation in the Rotated Arc Mixer (RAM).

\subsection{Compact model}

The compact model leans on the existence of a so-called "spectral decomposition" of the temperature evolution for the base flow $\mathbf{v}_{1}$ into elementary states (denoted "eigenmodes") according to

$$
\widetilde{T}(\mathbf{x}, t)=\sum_{m=0}^{\infty} \alpha_{m} \phi(\mathbf{x}) e^{\lambda_{m} t}, \quad \widetilde{T}(\mathbf{x}, 0)=\sum_{m=0}^{\infty} \alpha_{m} \phi(\mathbf{x})
$$

with $\left\{\phi_{m}, \lambda_{m}\right\}$ the eigenfunction-eigenvalue pairs defined by the eigenvalue problem

$$
P e^{-1} \nabla^{2} \phi_{m}-\mathbf{v}_{1} \cdot \nabla \phi_{m}=\lambda_{m} \phi_{m}
$$

corresponding with (6) and $\alpha_{m}$ the expansion coefficients determined by the initial condition [7, 10]. Progressive decay of transient temperatures towards a bound implies stable eigenmodes $\left(\operatorname{Re}\left(\lambda_{m}\right)<0\right)$ that are ordered according to decay rate $\ldots \leq \operatorname{Re}\left(\lambda_{1}\right) \leq R e\left(\lambda_{0}\right)<0$. Unsteady flow composition from piece-wise steady Stokes flows, following (5), in conjunction with boundary conditions, as per (7), transfers relation (4) between flow $\mathbf{v}_{k}$ and base flow $\mathbf{v}_{1}$ to the thermal regime governed by (6). Thus, spectral temperature evolution for $\mathbf{v}_{k}$ is implies a reorientation of 13 following

$$
\widetilde{T}(\mathbf{x}, t)=\sum_{m=0}^{\infty} \alpha_{m}^{(k)} \phi_{m}\left(\mathcal{R}_{k}(\mathbf{x})\right) e^{\lambda_{m} t}, \quad \widetilde{T}(\mathbf{x}, 0)=\sum_{m=0}^{\infty} \alpha_{m}^{(k)} \phi_{m}\left(\mathcal{R}_{k}(\mathbf{x})\right),
$$

comprising of an identical eigenvalue spectrum and a reorientation of the eigenfunctions of the base flow consistent with (4). Semi-analytical solution (10) has the same structure as spectral decompositions (13) and (15) and constitutes their discrete approximation on the computational grid $\mathbf{X}$, i.e.

$$
\widetilde{\mathbf{T}}(t)=\mathbf{V}_{k} e^{\boldsymbol{\Lambda} t} \mathbf{V}_{k}^{-1} \mathbf{T}_{0}=\sum_{m=0}^{M} \alpha_{m}^{(k)} \boldsymbol{\phi}_{m}^{(k)} e^{\lambda_{m} t}, \quad \boldsymbol{\alpha}^{(k)}=\mathbf{V}_{k}^{-1} \mathbf{T}_{0},
$$

with eigenvector $\boldsymbol{\phi}_{m}^{(k)}$ containing the nodal values of eigenfunction $\phi_{m}^{(k)}(\mathbf{x})$. Subscript "k" in $\boldsymbol{\Lambda}_{k}$ following (10) distinguishing an aperture-wise eigenvalue spectrum is omitted due to its invariance to 
reorientation according to (15). The eigenvector bases $\mathbf{V}_{k}$ relate to that of the base flow following

$$
\mathbf{V}_{k}=\mathbf{R}^{k-1} \mathbf{V}_{1}
$$

with rotation matrix $\mathbf{R}$ the discrete counterpart to operator $\mathcal{R}_{k}$ on the computational grid. This affords two major reductions in computational effort compared to numerical treatment of (6) via conventional spatio-temporal discretisations. First, semi-analytical solution (16) enables simulation of the temperature evolution by a matrix-vector multiplication instead of a (computationally far more expensive) time-marching scheme. (Spectral decomposition and relevant matrices are constructed in a pre-processing stage.) Second, (16) enables simulations for apertures $k>1$ entirely in terms of the spectral decomposition of the base flow $(k=1)$ upon rotation of the eigenvectors via (17).

(a) $m=0$.

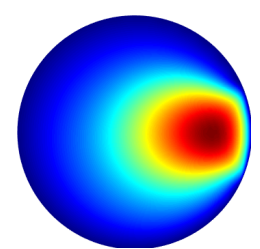

(e) $m=7(R e)$.

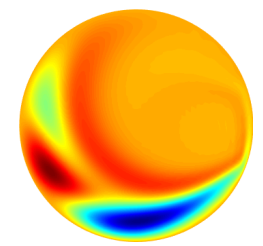

(b) $m=1$.

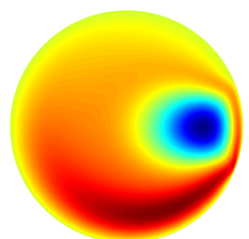

(f) $m=7(\operatorname{Im})$.

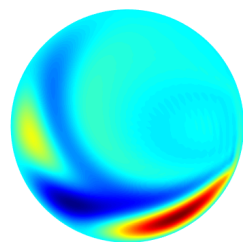

(c) $m=2(R e)$.

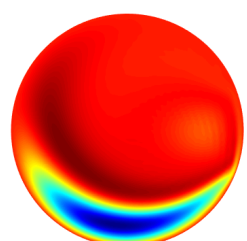

(g) $m=10(R e)$.

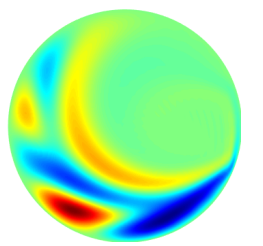

(d) $m=2(\operatorname{Im})$.

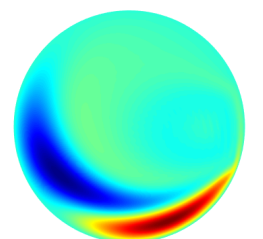

(h) $m=10(\operatorname{Im})$.

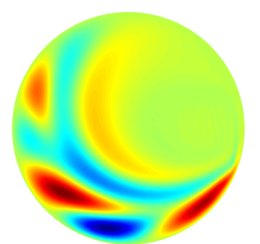

Figure 5 The leading dynamic modes of finite dimensional approximation of the advection diffusion matrix for the base flow $\mathbf{v}_{1}$ of an advection dominated flow $\left(P e=10^{3}\right)$.

Further reduction in computational effort exploits the exponential decay of each eigenmode $m$ in the spectral decomposition with characteristic time scale $\tau_{m}=-1 / \operatorname{Re}\left(\lambda_{m}\right)$. The 'slow' modes are negligible when $\tau_{m} / \tau \ll 1$ and thus admit an approximation of the temperature evolution by truncated expansion

$$
\widetilde{T}(t) \approx \widehat{\mathbf{T}}(t)=\sum_{m=0}^{Q} \widehat{\alpha}_{m}^{(k)} \boldsymbol{\phi}_{m}^{(k)} e^{\lambda_{m} t}, \quad \widehat{\boldsymbol{\alpha}}^{(k)}=\mathbf{G}_{k} \mathbf{T}_{0}, \quad \mathbf{G}_{k}=\left(\widehat{\mathbf{V}}_{k}^{\dagger} \widehat{\mathbf{V}}_{k}\right)^{-1} \widehat{\mathbf{V}}_{k}^{\dagger},
$$

with $Q \ll M$ and symbol . indicating approximate quantities, where the retained terns in the spectral decomposition define the so-called "dominant eigenmodes" [7, 10. Fig. 5 shows the leading dominant eigenmodes $0 \leq m \leq 10$ corresponding with the base flow $\mathbf{v}_{1}$. Note that expansion coefficients $\widehat{\boldsymbol{\alpha}}^{(k)}$ emanate from a least-squares projection of the initial condition on the truncated eigenvector basis $\widehat{\mathbf{V}}=$ $\left[\boldsymbol{\phi}_{1}^{(k)}, \ldots, \boldsymbol{\phi}_{Q}^{(k)}\right]$. The retained modes generically include only a small subset of the full set; application to a similar transport problem results in acceptable reductions of the model size and, inherently, the required computational effort by an order of magnitude, i.e. $Q / M \sim \mathcal{O}\left(10^{-1}\right)$ [11]. An estimate of the total reduction in computational effort by the truncation and the previous factors is given in Sec. 4.3 .

\subsection{Control strategy revisited}

Reformulation of approximation 18 in an efficient matrix-vector operation

$$
\widehat{\mathbf{T}}_{k}\left(t_{n+1}\right)=\widehat{\mathbf{U}}_{k} \widehat{\boldsymbol{\alpha}}_{0}, \quad \widehat{\mathbf{U}}_{k}=\widehat{\mathbf{V}}_{k} e^{\widehat{\Lambda} \tau}, \quad \widehat{\boldsymbol{\alpha}}_{0}=\mathbf{G}_{k} \mathbf{T}\left(t_{n}\right), \quad \widehat{\boldsymbol{\Lambda}}_{k}=\operatorname{diag}\left(\lambda_{0}, \ldots, \lambda_{Q}\right),
$$


enables fast prediction of the aperture-wise temperatures $T_{k}(\mathbf{x}, t)$ in step 1 and subsequent selection of the aperture yielding the highest heating rate in step 2 via the discrete counterpart

$$
\arg \min _{k} \widehat{J}_{k}\left(t_{n+1}\right), \quad \widehat{J}_{k}(t)=\sum_{m=1}^{M} \gamma_{m} \widehat{T}_{k}^{2}\left(\mathbf{x}_{m}, t_{n+1}\right),
$$

of minimization problem (12), with $\gamma_{m}$ weight factors incorporating e.g. area weighting of $\widetilde{T}\left(\mathbf{x}, t_{n+1}\right)$. The reduction in computational effort afforded by the compact model can be estimated upon comparison to a conventional numerical model based on temporal discretisation of $(9)$ into $\widetilde{\mathbf{T}}_{p+1}=\mathbf{S}_{k} \widetilde{\mathbf{T}}_{p}$, with $\mathbf{S}_{k}$ a system matrix constructed from $\mathbf{A}_{k}$ and $t_{p}=p \Delta t$ the time steps of the time-marching scheme. Prediction of the aperture-wise temperatures $\widetilde{T}_{k}$ by the compact model $(19)$ requires two consecutive matrix-vector multiplications with $Q \times M$ matrix $\mathbf{G}_{k}$ and $M \times Q$ matrix $\mathbf{U}_{k}$. A conventional scheme, on the other hand, typically necessitates $\tau / \Delta t=N \sim \mathcal{O}\left(10^{2}-10^{3}\right)$ steps for time interval $\tau=t_{n+1}-t_{n}$ that each require a matrix-vector multiplication with $M \times M$ matrix $\mathbf{S}_{k}$. Thus the compact and conventional method involve $c_{1}=4 Q M$ flops and $c_{2}=2 N M^{2}$ flops, respectively, per prediction. (Matrix-vector products with an $I \times J$ matrix involve $c=2 I J$ flops [12].) This yields $c_{2} / c_{1}=N M / 2 Q \sim \mathcal{O}\left(10^{3}-10^{4}\right)$ as relative computational effort, exposing the dramatic reduction by the compact model that makes the control strategy proposed in Sec. 4.1 viable for practical applications.

It must be stressed that, though the present study employs the dedicated approach by Ref. [10], system matrix $\mathbf{A}_{1}$ of the base flow and its spectral decomposition underlying the above compact model can be obtained by spatial discretization of (6) using conventional FDM/FVM/FEM schemes. Commercial simulation packages as e.g. COMSOL Multiphysics in fact conveniently provide said (FVMbased) system matrix via exportation from a standard CFD model. Moreover, reliance of the approach on only a small subset $Q \ll M$ of dominant eigenmodes permits their identification by dedicated numerical algorithms for determination of leading eigenvalue-eigenvector pairs of large matrices. The present study e.g. utilizes the built-in function eigs in MATLAB for this purpose.

Finally, so-called "Dynamic Mode Decomposition" (DMD) enables determination of the dominant eigenmodes - and construction of the compact model (19) - directly from temperature time series $\left\{\mathbf{T}\left(t_{0}\right), \mathbf{T}\left(t_{1}\right), \ldots, \mathbf{T}\left(t_{n}\right), \ldots\right\}$ on discrete grids $\mathbf{X}$. A major advantage of DMD is that it concerns a data-based technique and thus (in principle) facilitates the proposed control strategy using data from conventional CFD, laboratory experiments or field measurements [13. However, DMD often is sensitive to (experimental) noise, which may hamper isolation of sufficient dominant eigenmodes for a reliable approximation (18). (Compare e.g. the leading eigenmodes obtained from CFD versus experimental data in Ref. [7.) The model-based approach adopted in the present study is far more robust in this respect, but also more laborious than DMD. Hence both the model-based and data-based approach offer ways to realize the proposed control strategy yet their suitability and feasibility are case-specific.

\subsection{Performance}

Performance of the novel approach is compared to that of a time-periodic scheme designed for optimal mixing in Fig. 6. Here, adaptive schemes are employed that allow i) only clockwise boundary motion at each aperture $(\omega=1$ in (2) $)$ ii) or both clockwise and counter-clockwise boundary motion at each aperture $(\omega= \pm 1$ in (2p) during the minimization of (20). Note that flow reversal in the latter permits $2 N=6$ potential aperture candidates that can minimize $\hat{J}_{k}(t)$ in $(20)$ each time-step $t_{n}$ instead of $N=3$. A comparison of the adaptive scheme, without flow reversal, with a time-periodic scheme in Fig. 6 reveals faster homogenization from 250 to 210 non-dimensional time-units (16\% reduction). Flow reversal further accelerates this homogenization by shortening the transient from 250 to 185 time units (26\% reduction). Temperature snapshots for the adaptive scheme with and without flow reversal are shown in top and bottom row of Fig. 7 respectively. Comparison of these snapshots to those of the time-periodic scheme (Fig. 3) reveals an entirely different structure with hot plumes emerging from the 
(a) Schemes.

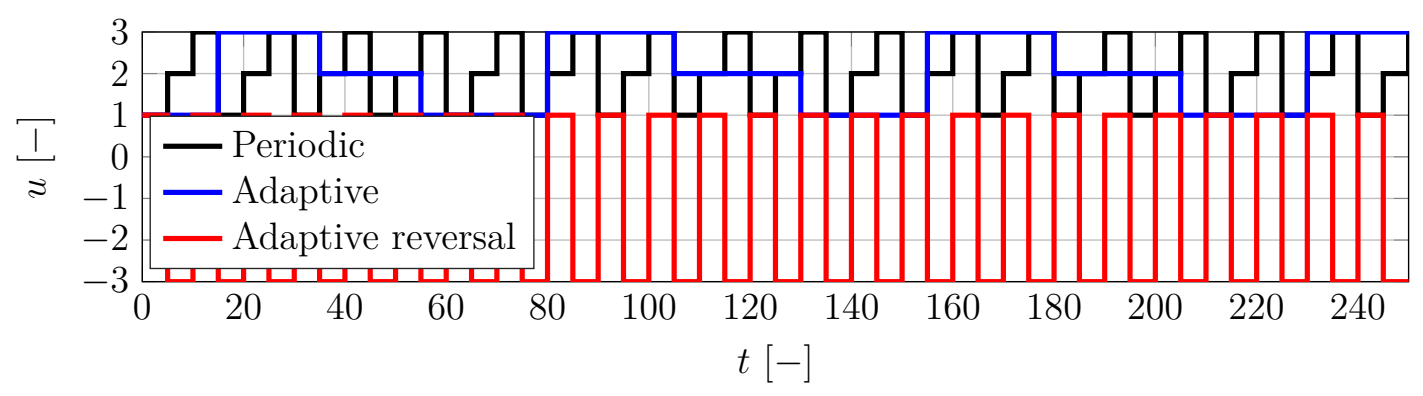

(b) Error.

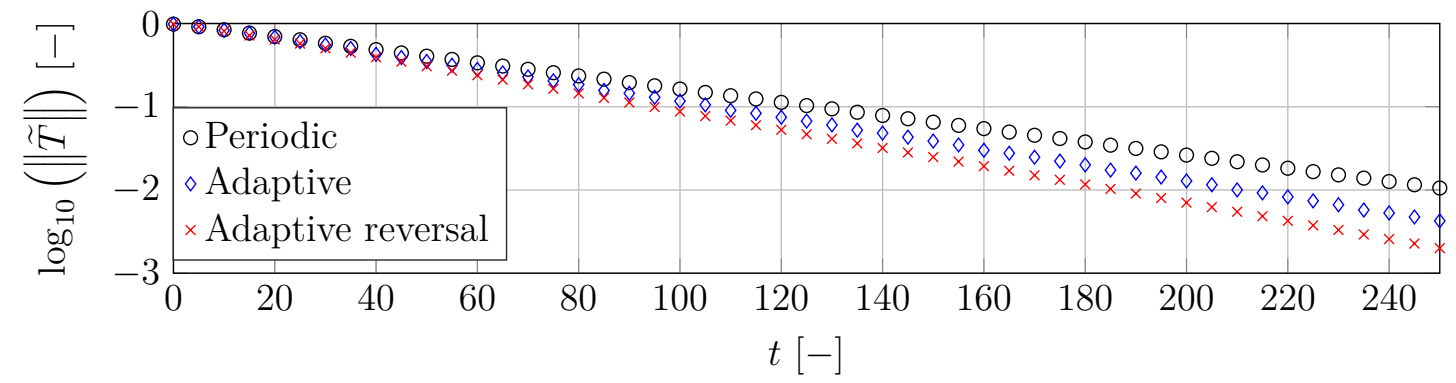

Figure 6 The (a) orientation scheme and (b) the transient temperature for the time-periodic and the novel schemes for advection dominated transport $\left(P e=10^{3}\right)$ with $\tau=5$.
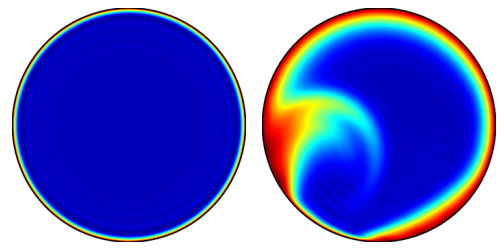

(b) $t_{n}=10$.
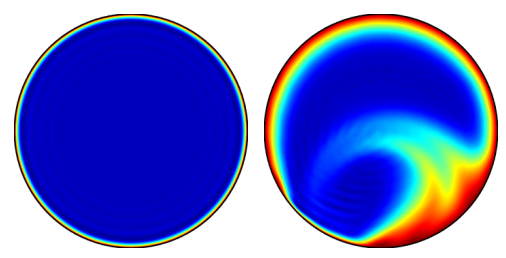
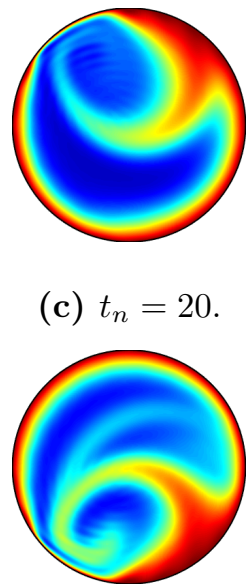

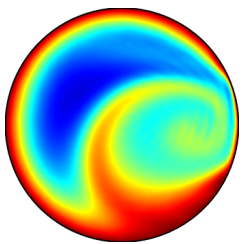

(d) $t_{n}=30$.

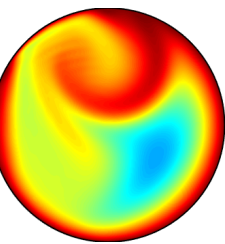

(e) $t_{n}=50$.
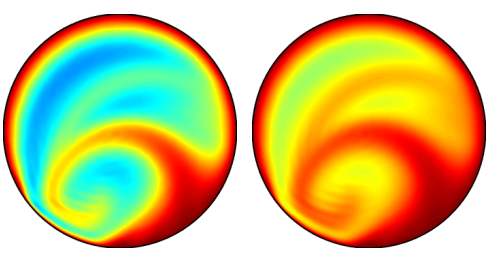

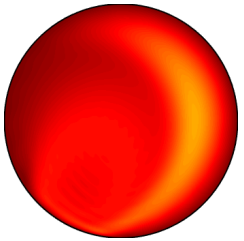

(f) $t_{n}=100$.

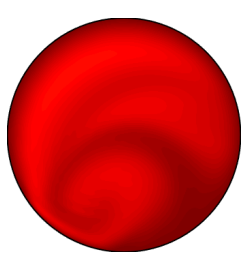

Figure 7 Temperature evolution $T(\mathbf{x}, t)$ of an advection dominated flow $\left(P e=10^{3}\right)$ with aperture activation time $\tau=5$ of the novel flow orientation scheme driven in only clockwise (top row) and in both clockwise and counter-clockwise directions (bottom row).

boundary for the adaptive approach. Minimization of 20 with flow reversal in fact yields a periodic switching between aperture $1(\omega=1)$ and $3(\omega=-1)$. These results demonstrate the great potential of the novel scheme compared to the time-periodic approach.

\section{CONCLUSIONS}

Conventional schemes for enhancement of scalar transport by flow reorientation consist of a periodic reorientation (in space or time) of the flow designed to accomplish efficient fluid mixing. This approach hinges on the assumption that efficient mixing automatically yields efficient scalar transport. The current study demonstrates by way of a representative heat-transfer problem, i.e. heating of an initially 
cold fluid via a hot boundary, that this premise not necessarily holds in case of scalar transport involving significant diffusion and exchange across the boundary. Adaptive reorientation of the flow based on predicted future evolutions of the temperature field from feedback of intermediate states by the proposed scheme namely substantially accelerates the heating of the cold fluid compared to conventional periodic schemes. A compact model (which can be constructed in various ways) enables fast predictions of the temperature evolution and thus makes the proposed scheme (in principle) applicable for real-time process control.

Experimental investigations of the performance of the adaptive scheme for the heating problem considered in this study are in progress. Future efforts aim at (further) paving the way to practical application of the proposed scheme by data-based construction of compact models, estimation of the full temperature field from discrete sensor data and development of control strategies for establishment of heterogeneous states (e.g. thermal fronts for promotion of chemical reactions).

\section{ACKNOWLEDGMENTS}

This research has been made possible by the Netherlands Organisation for Scientific Research (NWO) as part of the Computational Sciences for Energy Research Initiative (CSER; grant 15CSER15).

\section{References}

[1] H. Aref, J.R. Blake, M. Budišić, S.S.S. Cardoso, J.H.E. Cartwright, H.J.H. Clercx, K. El Omari, F. Ulrike, R. Golestanian, E. Gouillart, G.F. van Heijst, T.S. Krasnopolskaya, Y. Le Guer, R.S. MacKay, V.V. Meleshko, G. Metcalfe, I. Mezić, A.P.S. de Moura, O. Piro, M.F.M. Speetjens, R. Sturman, J.-L. Thiffeault and I. Tuval, Frontiers of chaotic advection, Reviews of Modern Physics, 89 (2017) 1-66.

[2] M. Speetjens, S. Varghese and R. Trieling, Lagrangian approach to analysis and engineering of two generic transport problems in enhanced subsurface flows, Journal of Contaminant Hydrology, 224 (2019) 1-22.

[3] A. N. Piscopo, R. M. Neupauer, D. C. Mays, Engineered injection and extraction to enhance reaction for improved in situ remediation, Water Resources Research, 49 (2013) 3618-3625.

[4] M. G. Trefry, D. R. Lester, G. Metclafe, A. Ord, K. Regenauer-Lieb, Toward enhanced subsurface intervention methods using chaotic advection, Journal of Contaminant Hydrology, 127 (2012) 15-29.

[5] J. M. Ottino, The kinematics of mixing: Stretching, chaos and transport, Cambridge University Press, Cambridge, 1989.

[6] V.S. Dolk, M. Lauret, D.J. Antunes, P.D. Anderson and W.P.M.H. Heemels, A switched system approach to optimize mixing of fluids, International Federation of Automatic Control, 51 (2018) 31-36.

[7] O. Baskan, M.F.M. Speetjens, G. Metcalfe and H.J.H. Clercx, Experimental and computational study of scalar modes in a periodic laminar flow, International Journal of Thermal Sciences, 96 (2015) 102-118.

[8] T.-Y Hwu, D.-Liang Young and Y.-Y. Chen, Chaotic advections for Stokes flows in circular cavity, Journal of Engineering Mechanics, 123 (1997) 774-782.

[9] H. K. Khalil, Nonlinear systems, Pearson Education, 2014.

[10] D.R. Lester, M. Rudman and G. Metcalfe and H.M. Blackburn, Global parameteric solutions of scalar transport, Journal of Computational Physics, 227 (2008) 3032-3057.

[11] O. Gorodetskyi, M.F.M. Speetjens and P.D. Anderson, An efficient approach for eigenmode analysis of transient distributive mixing by the mapping method, Physics of Fluids, 24 (2012) 053602.

[12] G. Strang, Linear Algebra and Its Applications, Thomson, Brooks/Cole, 2006.

[13] J. N. Kutz, S. L. Brunton, B. W. Brunton, J. L. Proctor, Dynamic Mode Decomposition: Data-Driven Modeling of Complex Systems, SIAM, 2016. 